EPJ Web of Conferences 60, 05004 (2013)

DOI: $10.1051 /$ epjconf/20136005004

(C) Owned by the authors, published by EDP Sciences, 2013

\title{
Heavy Flavour and Quarkonium Production at the LHC
}

\author{
Hermine K. Wöhri ${ }^{1}$, a for the ATLAS, CMS and LHCb collaborations \\ ${ }^{1} \mathrm{CERN} / \mathrm{PH}$, Geneva, Switzerland
}

\begin{abstract}
Recent results on heavy flavour and quarkonium production, obtained by ATLAS, CMS and LHCb using data from the 2011 and 2012 pp runs, are summarised. They include $\mathrm{J} / \psi, \psi^{\prime}$ and $\Upsilon$ cross sections and polarizations, as well as the first prompt X(3872) differential cross section. Results in the heavy flavour sector include an updated $b \bar{b}$ cross section measurement differential in the separation between two b-tagged jets. Some first measurements at $\sqrt{s}=8 \mathrm{TeV}$ are also shown.
\end{abstract}

\section{Quarkonium Production}

Heavy quarkonia are supposed to be ideal probes to study the non-perturbative process of hadron formation: given their heavy constituent quarks, these $Q \bar{Q}$ systems are expected to be characterized by non-relativistic velocities and by relatively small $\alpha_{S}$ values. The truth is, however, that quarkonium production is not yet understood: while the NRQCD framework [4] describes the measured quarkonium $p_{\mathrm{T}}$ spectra to a satisfactory level (freely adjusting the "long-distance matrix elements"), it fails to reproduce the polarization measurements. The LHC experiments [1-3] strongly contribute to this field of research, providing a wealth of high precision measurements, extending to hitherto unreached values of transverse momentum and rapidity.

\subsection{Conventional Quarkonium Production and Polarization}

Polarization measurements represent particularly stringent observables, as the theoretical calculations do not have free parameters to be adjusted from the data. On the other hand, they are also experimentally challenging as they request large data samples, a very good understanding of the detector, as well as a multi-dimensional approach to the physics problem [5]. Figure 1 shows the polarization anisotropy parameter $\lambda_{\vartheta}$ for $\Upsilon(\mathrm{nS})$, as a function of $p_{\mathrm{T}}$. Neither the CMS [6] nor the CDF [7] data show the strong polarization predicted by state-of-the-art calculations from NLO NRQCD. Among all the quarkonium states the $\psi^{\prime}$ is particularly interesting, as it does not experience feeddown from heavier quarkonia (the beauty feed-down can be subtracted thanks to the lifetime distribution). CMS has measured the $\psi^{\prime}$ polarization up to $50 \mathrm{GeV}$, in two rapidity bins [8] (Fig. 2). Similarly to the $\Upsilon$ case, no strong polarization is seen, contrary to the strong transverse polarization predicted by NRQCD.

\footnotetext{
a e-mail: hermine.woehri@cern.ch
}

Using the measured $\Upsilon(\mathrm{nS})$ polarization as an input to calculate corresponding detector acceptances, CMS provided an updated $\Upsilon(\mathrm{nS})$ cross-section measurement [9], integrating the full statistics collected at $\sqrt{s}=7 \mathrm{TeV}$, shown in Fig. 3 together with a previous CMS measurement using 2010 data [10] and with the corresponding measurement from ATLAS [11]. The LHC data extend to $p_{\mathrm{T}}$ regions where the NRQCD calculations should be very reliable. The lower panels of Fig. 3 show the $\Upsilon 2 \mathrm{~S} / 1 \mathrm{~S}$ and $3 \mathrm{~S} / 1 \mathrm{~S}$ ratios, as a function of rapidity and $p_{\mathrm{T}}$. The rapidity distribution has the same shape for all the three states, while there is a strong increase of the cross-section ratios with increasing $p_{\mathrm{T}}$ : a high- $p_{\mathrm{T}} \Upsilon(2 \mathrm{~S})$ has a higher cross section (relatively speaking) than a $\Upsilon(1 \mathrm{~S})$ with the same $p_{\mathrm{T}}$. At $p_{\mathrm{T}} \gg M(\Upsilon)$ the data indicate a flattening of the crosssection ratio.

$\mathrm{LHCb}$ is the first experiment to measure quarkonium cross sections in pp collisions at $8 \mathrm{TeV}$ [12] (Fig. 4). The results, based on data samples collected in only a few weeks, cover the forward rapidity range 2.0-4.5 [13].

\subsection{Associated $\mathrm{J} / \psi$ and $\mathrm{W}^{ \pm}$production at $\sqrt{s}=7 \mathrm{TeV}$}

A further possibility to test NRQCD predictions is to study quarkonium production in association with vector bosons. ATLAS made a first measurement of prompt $\mathrm{J} / \psi$ production associated with $\mathrm{W}$ production [14], where the "double parton scattering" process (DPS) -leading to an identical signature - is considered as background, evaluated using data and validated by looking at the opening angle between the prompt $\mathrm{J} / \psi$ and the $\mathrm{W}$ (Fig. 5-left). The cross-section ratio between the associated $\mathrm{J} / \psi-\mathrm{W}$ production and inclusive $\mathrm{W}$ production (Fig. 5-centre) has been compared to various theoretical predictions (Fig. 5-right). While the (statistical) uncertainties are still quite large, the measured cross-section ratio is roughly an order of magnitude larger than all the theoretical predictions. 

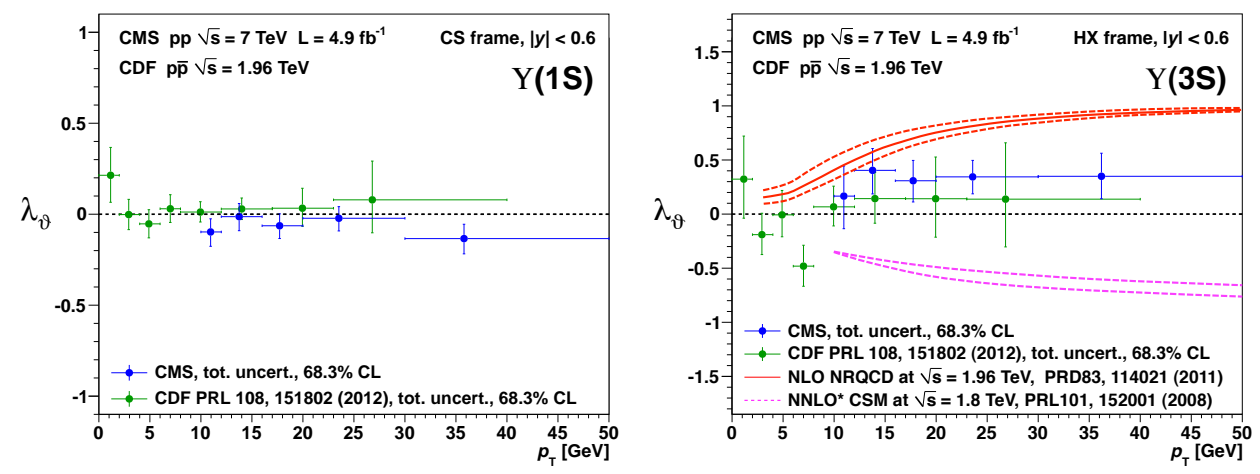

Figure 1. Polarization parameter $\lambda_{\vartheta}$ vs. $p_{\mathrm{T}}$ for $\Upsilon(1 \mathrm{~S})$ (left) and $\Upsilon(3 \mathrm{~S})$ (right), in the Collins-Soper (CS) and helicity (HX) frames, respectively, as measured by CMS [6] and CDF [7].

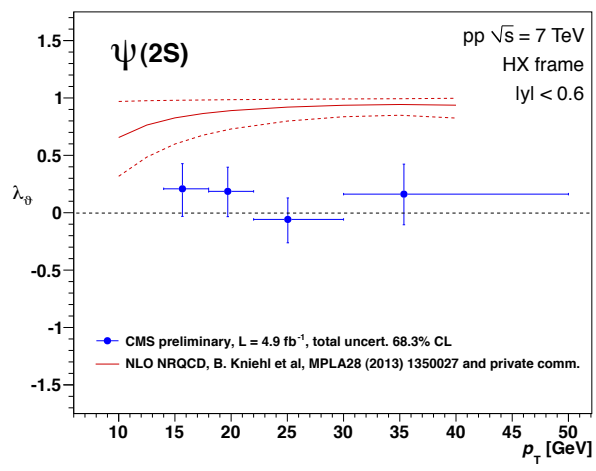

Figure 2. Polarization parameter $\lambda_{\vartheta}$ vs. $p_{\mathrm{T}}$ for the prompt $\psi^{\prime}$, for the mid-rapidity interval $|y|<0.6$, as measured by CMS [8].

\subsection{Exotic Quarkonium Production}

Since its discovery in 2003 by Belle, the properties of the $\mathrm{X}(3872)$ have been studied at the B-factories, the Tevatron and now at the LHC. CMS measured [15] its feed-down fraction from $\mathrm{B}$ decays, $26.3 \pm 2.3 \pm 1.6 \%$, practically independent of $p_{\mathrm{T}}$ (Fig. 6-left), as well as its prompt differential cross section (Fig. 6-centre), seen to be roughly a factor of 4 below the LO NRQCD predictions [16], tuned to reproduce the $p_{\mathrm{T}}$-integrated Tevatron data. The disagreement increases with $p_{\mathrm{T}}$. The measurement also provides the dipion mass distribution for $X(3872) \rightarrow J / \psi \pi \pi$ decays (Fig. 6-right), confirming that the decay is dominated by an intermediate $\rho$.

\section{Heavy Flavour Production}

The angular separation between two b-tagged jets has been measured by CMS [18] (Fig. 7), providing a test of perturbative QCD: at LO only a "back-to-back" emission of the two b-quarks is possible, while higher orders contribute through various processes ("gluon splitting", "flavour excitation", etc) to populate other topologies deviating from $\Delta \phi \sim \pi$. In the accessible phase space, reaching lower scales than previously studied [17], the distribution is still peaked at $\Delta \phi \sim \pi$, but there is a visible cross section extending down to $\Delta \phi \sim 0$. Calculations performed with MADGRAPH are the best at reproducing the observed shape, while PYTHIA describes best the overall normalization (Fig. 8).

The large cross sections of b quarks at LHC has enabled detailed studies of specific B hadrons. The most recent result comes from ATLAS, on $\mathrm{B}^{+}$production, extending the $p_{\mathrm{T}}$ coverage up to $120 \mathrm{GeV}$ [19]. The corresponding double-differential cross section is shown in Fig. 9-left. POWHEG, interfaced with Pythia for the hadronization process, describes rather well the doubledifferential shape, as well as the overall normalization, while MC@NLO (interfaced with Herwig) shows different trends: for $|y|<1$ the measured cross section is underpredicted and the simulations are too soft, while for $1.0<|y|<2.25$ they are too hard. The rapidity-integrated spectra (Fig. 9-centre) show good agreement with the previous CMS results [21]. FONLL calculations [20] show a slightly harder $p_{\mathrm{T}}$ spectrum, but otherwise describe the ATLAS data (Fig. 9-right).

From the previously mentioned quarkonium analysis at $\sqrt{s}=8 \mathrm{TeV}$, LHCb also extracted information on (inclusive) $\mathrm{B}$ hadrons by measuring non-prompt $\mathrm{J} / \psi$ 's from $B \rightarrow J / \psi X$ decays. The results are summarized in Fig. 10 . From LHCb's previous measurements $[22,23]$, the $p_{\mathrm{T}^{-}}$ and rapidity-integrated $\mathrm{B}$ hadron cross section (within $2.0<y<4.5$ and $p_{\mathrm{T}}<14 \mathrm{GeV}$ ) can be studied vs. $\sqrt{s}$. It shows a linear rise as a function of $\sqrt{s}$, increasing roughly by a factor of 3 when going from $\sqrt{s}=2.76$ to $8 \mathrm{TeV}$. The differential cross-section measurements have 

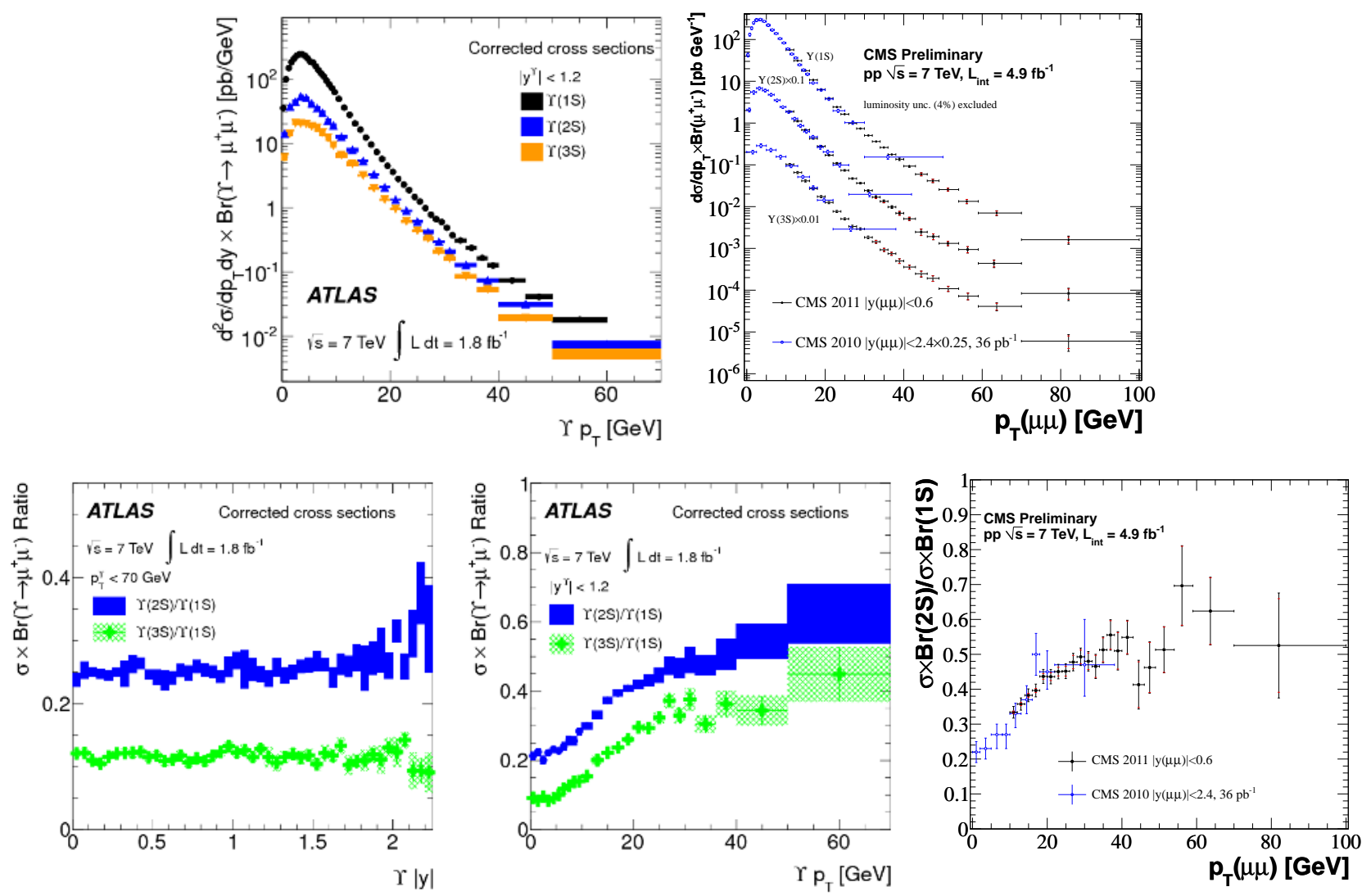

Figure 3. $\Upsilon$ cross sections (top) and ratios (bottom) as measured by ATLAS [11] (left) and CMS [9] (right).
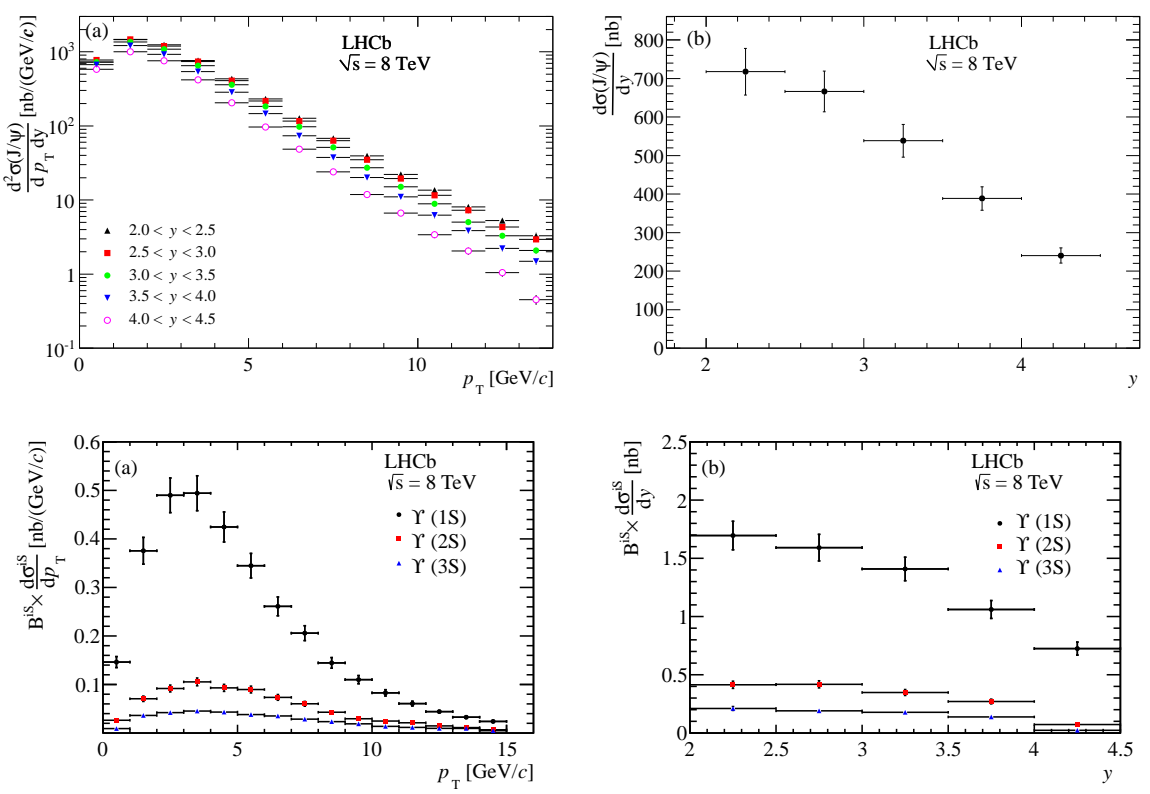

Figure 4. $\mathrm{J} / \psi$ (top) and $\Upsilon(\mathrm{nS})$ (bottom) cross sections vs. $p_{\mathrm{T}}$ (left) and $y$ (right), as measured by LHCb [12]. 

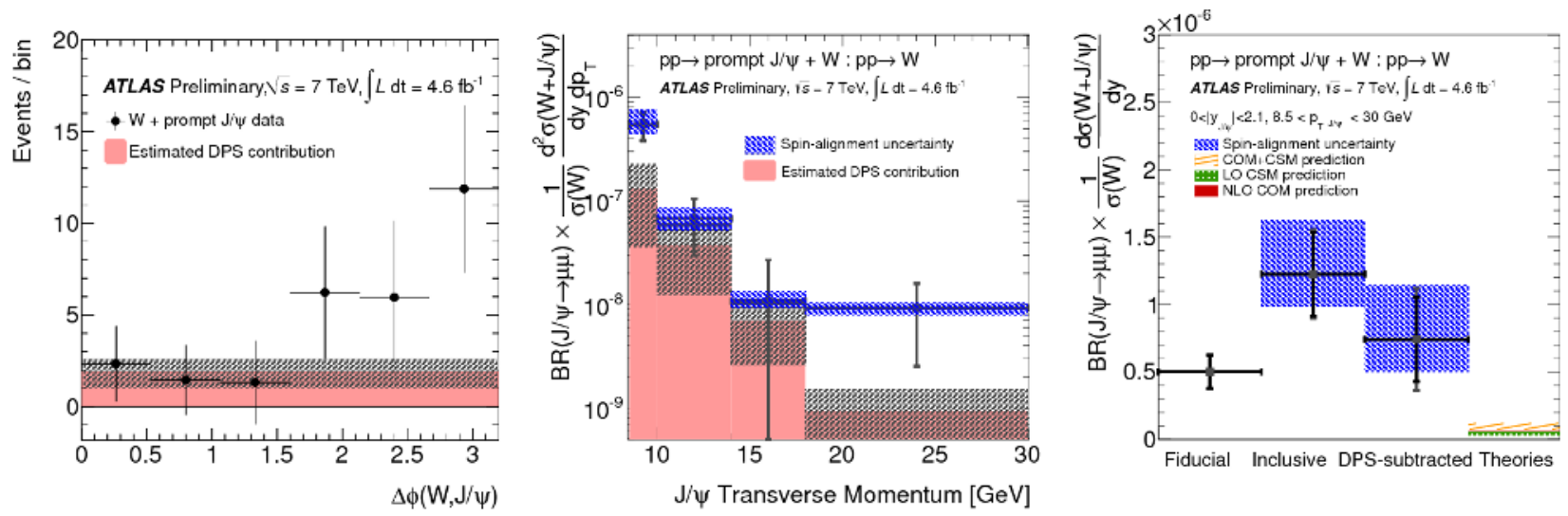

Figure 5. Associated prompt $\mathrm{J} / \psi$ and $\mathrm{W}$ production as measured by ATLAS [14]: angular separation between the $\mathrm{W}$ and $\mathrm{J} / \psi$ candidates (left); ratio to the inclusive $\mathrm{W}$ production cross section (centre); and comparison to theoretical predictions (right).
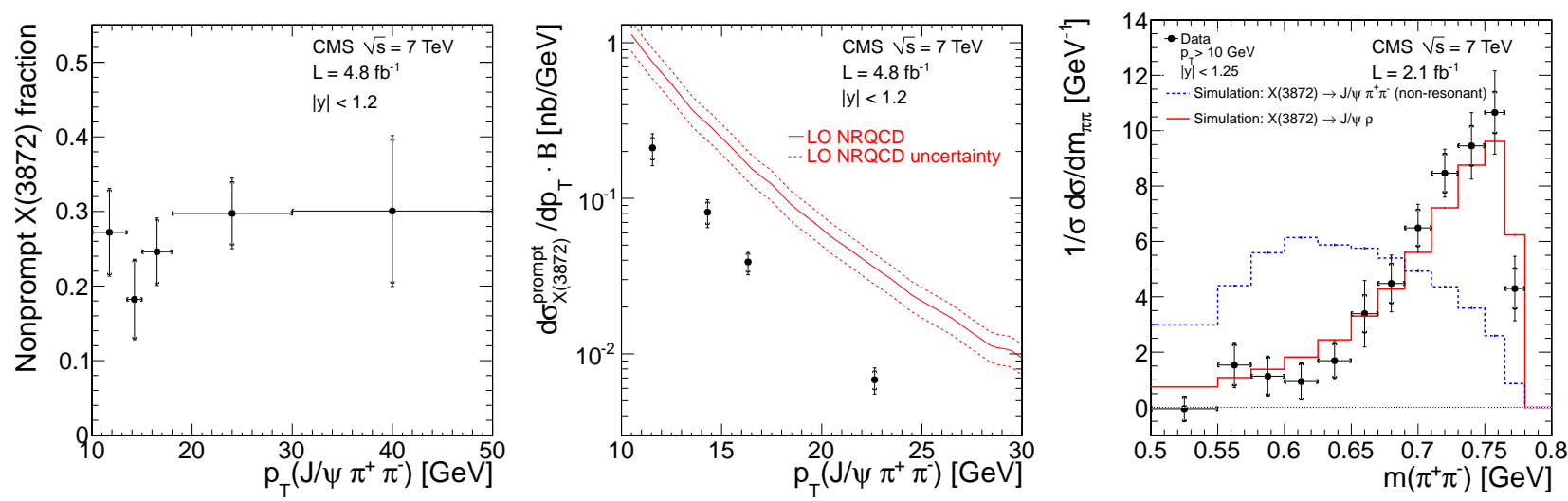

Figure 6. Fraction of $\mathrm{X}(3872)$ from $\mathrm{B}$ decays (left); prompt $\mathrm{X}(3872)$ cross section (centre); decay dipion mass distribution (right); as measured by CMS [15].
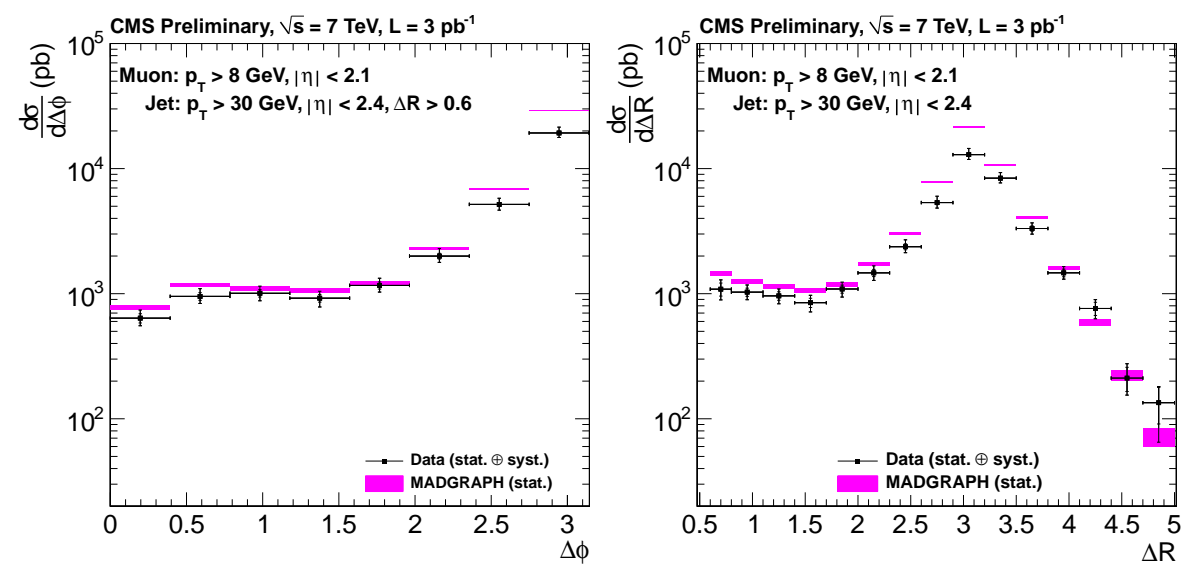

Figure 7. Beauty jet cross section as a function of $\Delta \phi$ (left) and $\Delta R$ (right), as measured by CMS [18] and compared to corresponding simulations with MADGRAPH. 

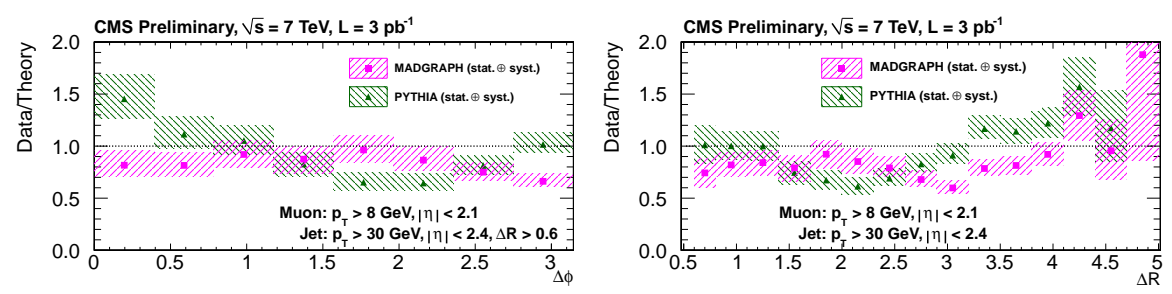

Figure 8. Comparison of the cross sections to MADGRAPH (magenta) and PYTHIA (green), after dividing the measurement by the theory calculations.
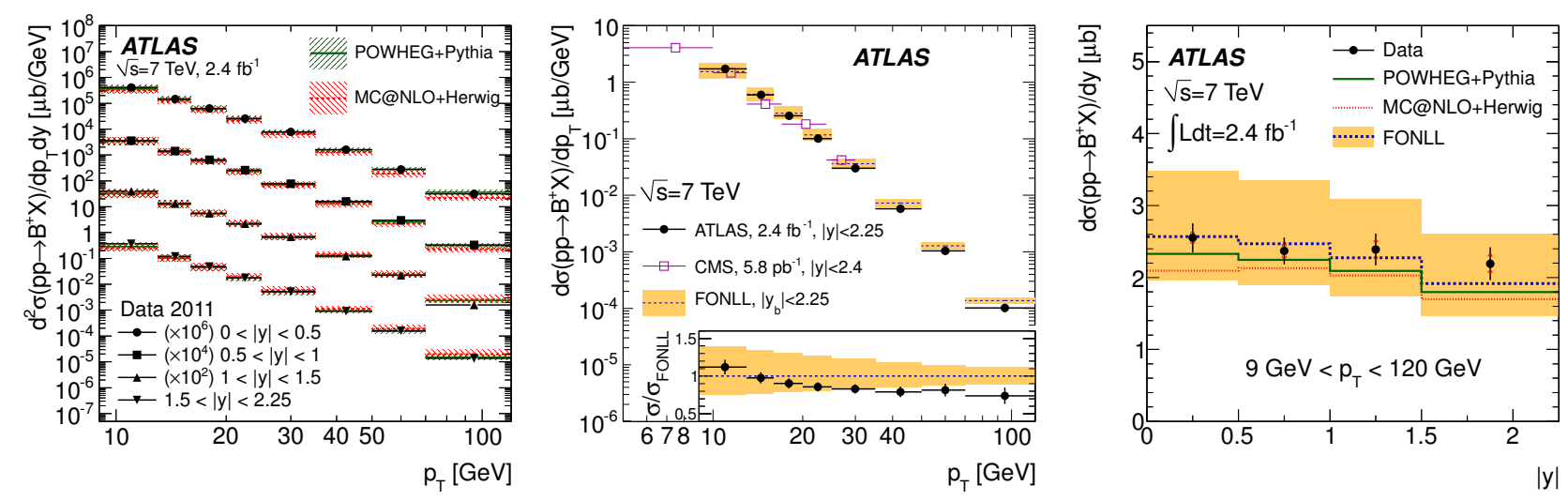

Figure 9. $\mathrm{B}^{+}$production as measured by ATLAS [19]: vs. $p_{\mathrm{T}}$ in several $y$ slices (left); vs. $p_{\mathrm{T}}$, integrated over $|y|<2.25$ and compared to FONLL calculations [20] (centre); vs. $y$, integrated within $9<p_{\mathrm{T}}<120 \mathrm{GeV}$.

been compared to FONLL [20] calculations and show very good agreement in all the three variables, giving confidence that these calculations can be trusted when making predictions for (differential) cross sections at even higher beam energies.

\section{References}

[1] ATLAS Coll., JINST 3 (2008) S08003.

[2] CMS Coll., JINST 3 (2008) S08004.

[3] LHCb Coll., JINST 3 (2008) S08005.

[4] G.T. Bodwin, E. Braaten and G.P. Lepage, Phys. Rev. D51, 1125 (1995)

[5] P. Faccioli, C. Lourenço, J. Seixas and H.K. Wöhri, Eur. Phys. J. C69, 657 (2010)

[6] CMS Coll., Phys. Rev. Lett. 110, 081802 (2013).

[7] CDF Coll., Phys. Rev. Lett. 108, 151802 (2012).

[8] CMS Coll., CMS-BPH-13-003, arXiv:1307.6070.
[9] CMS Coll., CMS-PAS-BPH-12-006.

[10] CMS Coll., arXiv:1303.5900, submitted to PLB.

[11] ATLAS Coll., Phys. Rev. D87, 052004 (2013).

[12] LHCb Coll., JHEP 06 (2013) 064.

[13] M. Pepe Altarelli, these proceedings and arXiv:1307.1110.

[14] ATLAS Coll., ATLAS-CONF-2013-042.

[15] CMS Coll., JHEP 04 (2013) 154.

[16] P. Artoisenet and E. Braaten, Phys. Rev. D81, 114018 (2010).

[17] CMS Coll., JHEP 03 (2011) 136.

[18] CMS Coll., CMS-PAS-BPH-10-019

[19] ATLAS Coll., arXiv:1307.0126, submitted to JHEP.

[20] M. Cacciari et al., JHEP 10 (2012) 137.

[21] CMS Coll., Phys. Rev. Lett. 106, 112001 (2011)

[22] LHCb Coll., JHEP 02 (2013) 041.

[23] LHCb Coll., Eur. Phys. J. C71 (2011) 1645. 
EPJ Web of Conferences
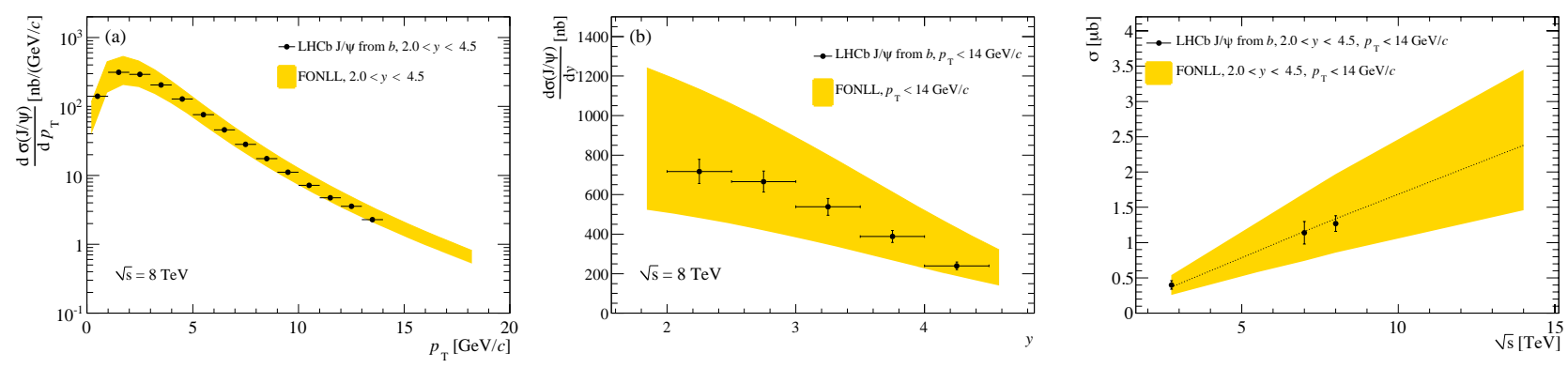

Figure 10. $\mathrm{J} / \psi$ production from $\mathrm{B}$ hadron decays, as a function of $p_{\mathrm{T}}$ (left), rapidity (centre) and beam energy (right), as measured by LHCb [12] and as calculated by FONLL [20]. 